\title{
Le mépris comme réassurance sociale
}

\section{Une petite bourgeoisie culturelle confrontée à son déclin}

\section{V3 Sociétés Contemporaines}

Elie Guéraut - elie.gueraut@gmail.com

\section{RESUME}

Cet article s'appuie sur les résultats d'une enquête ethnographique menée dans une ville moyenne en déclin du centre de la France. Il revient plus particulièrement sur la fragilisation de la petite bourgeoisie culturelle locale sous les effets conjugués des politiques nationales d'austérité, de la crise du socialisme municipal et du déclin urbain qui gagne les quartiers centraux. Dans ce contexte, les membres de ce milieu d'interconnaissance tentent d'exercer le pouvoir dont ils disposent encore afin de disqualifier les groupes sociaux qui lui contestent leur légitimité : la bourgeoisie économique locale, au pouvoir municipal depuis 2014, et les classes populaires résidant dans le centre-ville, dont le nombre progresse depuis la fin des années 2000. Le mépris de classe manifesté à l'égard de ces deux groupes permet alors une réassurance sociale collective de la petite bourgeoisie culturelle locale, qui tente ainsi de résister aux processus conjoncturels et structurels qui menacent son existence.

\section{ABSTRACT \\ Contempt as social reinsurance. A cultural petty bourgeoisie facing its decline}

This article is based on the results of an ethnographic survey conducted in a declining medium-sized city in central France. It analyses the weakening of the local cultural petty bourgeoisie under the combined effects of national austerity policies, the crisis of municipal socialism and the urban decline. In this context, the members of this group are trying to exercise the symbolic power they still have in order to disqualify the social groups that question their legitimacy: the local economic bourgeoisie, which has been in municipal power since 2014, and the working classes residing in the city centre, whose numbers have been increasing since the late 2000 s. The class contempt shown towards these two groups then allows a collective social reinsurance of the local cultural petty bourgeoisie, which thus tries to resist the cyclical and structural processes that threaten its existence.

\section{Introduction}

«Mais c'est qui ces beaufs ? Je vais leur en coller une !». Cette phrase, prononcée par une cadre de la mairie de Lergnes ${ }^{1}$ d'une quarantaine d'années, avant qu'elle ne quitte la salle et ses amis (et/ou collègues) en pleurs, vise un groupe de jeunes hommes situé à quelques mètres. Cheveux peignés en brosse et cols de leurs polos de marque relevés, ils se réjouissent à grands cris de la défaite de la liste d'union de gauche qui vient d'être annoncée. Nous sommes alors le 30 mars 2014, le soir du deuxième tour des élections municipales. Pour la première fois depuis 1965, le PS (Parti Socialiste) s'incline au profit de la coalition de trois listes, la principale «sans étiquette », rejointe entre les deux tours par l'UMP (Union pour un Mouvement Populaire) et l'UDI (Union Démocrate Indépendante). Plusieurs centaines de personnes sont venues suivre cette soirée électorale à l'issue incertaine dans la grande salle de réception de la mairie alors comble. Le petit groupe de personnes avec lequel j'attends les résultats, constitué de cadres de la commune et d'une élue socialiste (également directrice d'une association culturelle), n'y croyait de toute façon plus ; voilà près d'une heure que les mauvaises nouvelles en provenance des bureaux de vote s'accumulent. L'explosion de joie de

\footnotetext{
${ }^{1}$ Afin de préserver l'anonymat des enquêtés, le nom de la ville a été changé.
} 
ce groupe d'hommes, de même que la violence des propos de cette femme qui lui fait écho, sont ainsi à replacer dans l'intensité sociale de la scène, d'une importance rare du fait de la coprésence de groupes rivaux et de l'enjeu considérable que représente cette élection pour les protagonistes. Se jouent alors non seulement l'ostentation d'opinions partisanes, mais surtout une tension des frontières sociales qui séparent dans l'espace de cette salle les personnes en petits groupes affinitaires : ces jeunes, dont tout dans leur apparence laisse supposer une appartenance aux fractions économiques (inférieures) des classes moyennes, ou tout au moins une aspiration à s'y diriger, et ces quelques cadres de la FPT (Fonction Publique Territoriale) et cette élue, par ailleurs tous militants associatifs de longue date et membres de la petite bourgeoisie culturelle locale (cf. encadré). Le fait que soit employé le terme de « beaufs » n'a ainsi rien d'anodin compte tenu de la situation et des caractéristiques sociales des protagonistes. En associant ces jeunes à cette catégorie du sens commun teintée de mépris de classe et aux représentations sociales qu'elle charrie, l'enjeu est bien de qualifier négativement une altérité perçue comme indésirable et menaçante, et ainsi de participer à la réassurance d'un groupe dont la fragilisation vient ici de franchir une nouvelle étape.

Si cette scène revêt un caractère particulier pour les raisons que l'on a exposées, ce mode de production et d'énonciation du mépris de classe, comme instrument de disqualification et de réassurance sociale collective, ne lui est pas exclusif. En effet, la défaite électorale s'inscrit dans un processus plus large de fragilisation de la petite bourgeoisie culturelle, face auquel ses membres réagissent en affichant ostensiblement leur mépris à l'égard des groupes sociaux qui incarnent à leurs yeux ce déclin, en particulier cette (petite) bourgeoisie économique. Après avoir bénéficié du vaste mouvement d'externalisation d'emplois publics vers les collectivités (Biland, 2011, p. 19-29) et les associations (Hély, 2009, p. 3) dans les années 1980 et 1990, la petite bourgeoisie culturelle locale, composée de salariés directs et indirects de la «main gauche de l'Etat» (Bourdieu, 1993, p. 221-223) et de militants associatifs et politiques, fait face depuis les années 2000 à une politique systématique de réduction des dépenses publiques, qui lui porte préjudice sur les scènes professionnelle, associative et politique. Dès lors, les ressorts du mépris de classe que la petite bourgeoisie culturelle adresse aux groupes rivaux se doivent d'être analysés en considérant les tensions structurelles qui dépassent l'espace local: les transformations de l'Etat (contractualisation et précarisation de l'emploi public) et, plus largement, de la structure de l'espace social.

Pour autant, certaines caractéristiques du terrain d'enquête, une ville moyenne en déclin du centre de la France, participent à redoubler les effets de ces transformations, en particulier la forte dépendance de cet espace aux redistributions directes et indirectes de $1^{\prime}$ Etat $^{2}$. Cet état de fait n'est pas étranger à l'accélération des dynamiques de décroissance ${ }^{3}$ et de paupérisation qui s'observent à Lergnes depuis la fin des années $2000^{4}$ ainsi qu'au sein de

\footnotetext{
${ }^{2}$ Les plus gros employeurs de la zone d'emploi sont, par ordre décroissant d'importance, le centre hospitalier (1 900 emplois), les collèges et les lycées ( 850 emplois), le conseil général (850 emplois) et la commune de Lergnes (800 emplois) (source : INSEE, Siasp 2012).

${ }^{3}$ Le nombre d'habitants passe ainsi de près de 45000 en 1975 à environ 34000 en 2014 (sources : INSEE, RP 1975 et 2014).

${ }^{4}$ Le taux de chômage des 25-54 ans dans l'unité urbaine lergnoise était de 2,4 \% en 1968 et de 15,6 \% en 2013, contre respectivement 1,8\% et 11,6\% en France. Sources : Insee, RP 1968 et 2013.
} 
nombreuses autres villes moyennes françaises (Cauchi-Duval, Béal et Rousseau, 2016 ; Léger, 2011). Saisie dans ce contexte, la petite bourgeoisie culturelle lergnoise se distingue des «gentrifieurs » parisiens (Clerval, 2008, p. 132-133), lyonnais ou montreuillois (Collet, 2015), ou même de la «bourgeoisie progressiste » du quartier du South End à Boston (Tissot, 2011), en raison de son incapacité à lutter contre le déclin économique et symbolique des quartiers centraux de Lergnes où ses membres résident, en dépit d'un intense travail d'appropriation et de réhabilitation mené depuis les années 1980. Au total, la spécificité de cette petite bourgeoisie culturelle locale tient à la fragilisation qui s'exerce sur elle dans la période contemporaine, en raison du déclin du socialisme municipal, du désengagement de l'Etat, ou bien encore de cette perte de contrôle sur son espace résidentiel.

L'enquête ethnographique menée à Lergnes (cf. encadré) a permis de saisir cette fragilisation dans sa dimension relationnelle et localisée, c'est-à-dire de comprendre comment celle-ci s'inscrit dans les relations conflictuelles qu'entretient la petite bourgeoisie culturelle lergnoise aux groupes sociaux rivaux (ou jugés comme tels): la (petite) bourgeoisie économique lergnoise, au pouvoir municipal depuis mars 2014, ainsi que les classes populaires précarisées résidant dans le centre-ville. En dépit de la rareté des conflits interpersonnels, les deux groupes font l'objet d'un «commérage réprobateur » (Elias, 1985) intensif, qui vise à les associer à deux catégories du sens commun teintées de mépris de classe, celle de «beaufs » pour les premiers et de «cas soc' » (cas sociaux) pour les seconds. Si la petite bourgeoisie culturelle lergnoise, en tant que groupe chargé de relayer un ordre culturel légitime dans un «espace social localisé » (Laferté, 2015), n’a bien sûr pas le monopole de l' « ethnocentrisme de classe ${ }^{5}$, celui-ci se manifeste dans son cas avec force et intensité. C'est plus précisément l'absence d'euphémisation ou de dissimulation du mépris de classe qui le distingue, comme si les dispositions éthiques à se dire et/ou se faire l'allié des dominés, et plus généralement à produire un discours empathique sur le monde social, avaient été neutralisées.

Quels sont, pour un groupe social dominant mais fragilisé, les modes de perception et d'énonciation d'une altérité sociale jugée indésirable ? Il s'agit ici de montrer que l'emploi du registre du mépris de classe procède de la déstabilisation de la petite bourgeoisie culturelle et s'exerce différemment selon qu'il cible «la droite » (la petite bourgeoisie économique) ou «le bas » (les classes populaires précarisées) de l'espace local. Après avoir caractérisé la fragilisation qui frappe la petite bourgeoisie culturelle lergnoise, je montrerai que ce mépris de classe peut se faire instrument de «pouvoir symbolique », en ce qu'il fonctionne comme un rappel à l'ordre des choses, ayant pour effet immédiat d'imposer un sentiment d'illégitimité aux membres de la petite bourgeoisie économique locale (Bourdieu, 1977, 2016, p. 139-148). Mais il est aussi outil de compensation des positions sociales et de la légitimité perdues face aux classes populaires précarisées, qui chaque jour semblent davantage « donner le ton » (Chamboredon et Lemaire, 1970, p. 6-7) au sein des quartiers de centre-ville.

\footnotetext{
${ }^{5}$ Il en est par exemple ainsi de cette bourgeoisie progressiste étudiée par Sylvie Tissot, qui, bien que défendant dans les discours et dans les faits la «diversité » de leur quartier, distinguent une altérité désirable d'une altérité indésirable, tout en définissant les modalités de leur cohabitation avec les classes populaires. On peut en dire tout autant des nouvelles générations de gentrifieurs du bas Montreuil, qui, si elles déclarent apprécier la «mixité », ne la recherchent néanmoins plus dans les actes comme le faisaient leurs aînés, se justifiant par « fatalisme sociologique ».
} 
UNE ENQUETE ETHNOGRAPHIQUE DANS UNE VILLE MOYENNE EN DECLIN

Cet article s'appuie sur des matériaux collectés dans le cadre d'une enquête ethnographique au long cours (2010-2018) dans une ville moyenne en déclin du centre de la France (Guéraut, 2018). La thèse qui en est tirée interroge la crise de la reproduction d'un groupe social particulier, la petite bourgeoisie culturelle lergnoise, un milieu d'interconnaissance dont les membres occupent des positions sociales fondées sur la détention de capital culturel (professeurs, éducateurs, artistes, cadres de la FPT, salariés associatifs, etc.). Cet aspect rapproche la petite bourgeoisie culturelle lergnoise de la «petite bourgeoisie nouvelle »; les deux groupes partagent en effet une tendance à investir des « formes de culture qui sont, au moins provisoirement, aux marges (inférieures) de la culture légitime » avec une «intention évidente de réhabilitation» (Bourdieu, 1979, p. 417). Mais la notion de «petite bourgeoisie culturelle » vise aussi à qualifier la position particulière qu'occupe le groupe au sein de l'espace social localisé lergnois, celle d'un dominant dominé. Si ses membres y exercent une certaine autorité culturelle, en particulier envers les moins dotés en capital culturel - les classes populaires résidant dans le centre-ville (ouvriers, employés et inactifs) et la petite bourgeoisie économique (cadres et professions intermédiaires du secteur privé, artisans et commerçants, petits chefs d'entreprise) -, ils restent subordonnés à une bourgeoisie locale mieux (et plus anciennement) établie (maires et députés, professions libérales, patrons des principales entreprises locales, directeurs d'institutions publiques).

Cette enquête emploie les outils habituels de l'ethnographie : la réalisation d'entretiens (96 entretiens avec 62 personnes différentes), la consultation d'archives (personnelles, d'associations, des collectivités et des institutions publiques locales), l'observation directe et participante, notamment au sein d'un grand nombre d'associations culturelles, mais aussi de plusieurs organisations patronales. J'ai également analysé les données du recensement de la population (RP) produites par l'Insee, à l'échelle infracommunale (IRIS), de manière à rendre compte des dynamiques de paupérisation et de décroissance dans les différents quartiers de Lergnes, mais aussi à l'échelle des communes et des unités urbaines (UU), dans l'objectif d'appréhender le phénomène de déclin des villes moyennes sur l'ensemble du territoire métropolitain.

\section{Quand l'Etat fait et défait un groupe social}

Dans les années 1980, sous l'impulsion des politiques culturelles Lang et des lois de décentralisation, les effectifs des professions culturelles (Moulinier, 2016, p. 93-96 ; Urfalino, 2010, p. 310-328), et plus généralement ceux de la main gauche de l'Etat, augmentent considérablement en France (Pouget, 2005). Lergnes n'échappe pas à la tendance ; à la faveur de ce contexte, une génération de jeunes militants associatifs nés au début des années 1960 parvient en moins d'une décennie à exercer un quasi-monopole sur la création et la diffusion de biens culturels. Ils s'imposent d'abord sur la scène associative locale, en important des grandes agglomérations où ils poursuivent des études supérieures des biens symboliques issus des «marges inférieures de la légitimité culturelle», alors en voie de consécration (Coulangeon, 2010, p. 9-10) : une association organisant des expositions d'art contemporain 
en 1983, un festival de courts-métrages en 1984, de musique rock en 1986, de jazz en 1987. Toutes ces associations se professionnalisent et s'institutionnalisent dans les années 1990, jusqu'à former dans les années 2000 de véritables «entreprises associatives marchandes » (Hély, 2009, p. 113-115) comptant de nombreux salariés, toujours dirigées et présidées par les membres de cette génération de militants. Un grand nombre d'entre eux entreprend par ailleurs une «reconversion militante» (Tissot, Gaubert et Lechien, 2005), certains à destination des collectivités, faisant de la «culture » et des «quartiers » leurs catégories d'action publique privilégiées, d'autres vers le champ politique local sous la bannière socialiste. Un de ces jeunes militants associatifs devient élu municipal délégué aux affaires culturelles de 1989 à 1995. Il est rapidement rejoint par plusieurs de ses camarades ; ils seront deux à siéger au Conseil municipal après les élections municipales de 1995, cinq après celles de 2001, et six après celles de 2008. A l'aube des années 2000, cette petite bourgeoisie culturelle atteint alors son apogée : plusieurs de ses membres vivent exclusivement de leurs mandats d'élu et/ou occupent des postes de direction dans les collectivités et les structures parapubliques, les associations culturelles créées trente ans plus tôt occupent alors une position locale hégémonique et obtiennent des montants records de subventions ${ }^{6}$.

Les années 2000 marquent le début de la fragilisation de la petite bourgeoisie culturelle lergnoise en raison d'une accélération du désengagement de l'Etat qui se joue alors et dont la RGPP (Révision Générale des Politiques Publiques) de 2007 est emblématique (Dreyfus, 2011). Ces réformes structurelles, qui visent à réduire les dépenses publiques et le nombre de fonctionnaires au sein des institutions relevant directement de l'Etat, ne sont, sur le principe, pas supposées produire d'effets sur les services décentralisés. Pourtant, nombreux sont les sociologues et politistes à voir derrière la RGPP, un «réinvestissement des territoires » par l'Etat (Aust et Cret, 2012 ; Epstein, 2015), dont l'un des objectifs est bien de mettre à la diète les collectivités territoriales. Ce mouvement se traduit dans les années 2000 par une réévaluation à la baisse des objectifs des politiques culturelles (Fondu et Vermerie, 2016 ; Négrier et Teillet, 2013) et de la politique de la ville (Epstein, 2011), deux catégories d'action publique autour desquelles s'est construite l'ascension de la petite bourgeoisie lergnoise. Ce mouvement est palpable à travers l'examen de la trajectoire économique de ces associations culturelles fondées dans les années 1980 qui, toutes, voient le montant des subventions attribuées par l'Etat et les collectivités baisser durant la décennie, obligeant plusieurs d'entre elles à licencier une partie de leurs salariés. Ce désengagement de l'Etat contribue également à fragiliser la FPT, principal employeur de la zone d'emploi et « filière de promotion sociale» (Gollac et Hugrée, 2015) privilégiée des membres de la petite bourgeoisie culturelle ${ }^{7}$, de sorte que les générations nées dans les années 1970, et plus encore 1980, parviennent avec bien moins de succès que celle née dans les années 1960 à y opérer une reconversion militante pérenne ${ }^{8}$.

\footnotetext{
${ }^{6}$ Cumulées, ces subventions peuvent alors dépasser $400000 €$ par an (en euros constants de 2016) pour une seule association durant la décennie (sources : archives associatives et personnelles).

${ }^{7}$ Parmi les 98 personnes dont la trajectoire a pu être reconstruite pour les besoins de cette enquête, on compte 24 salariés de la FPT (dont 15 en catégorie A, 6 en catégorie B et 3 en catégorie C), 14 professeurs et instituteurs, 9 salariés d'association (dont 3 directeurs et 6 employés).

${ }^{8}$ Les plus jeunes, moins nombreux, sont aussi les plus sujets aux contrats précaires, à plus forte raison au sein des filières culturelles. Dans les trois principales collectivités de la zone d'emploi, le taux de non-titulaires atteint
} 
Le cas de Lergnes vient ainsi faire écho au constat dressé par d'autres : la réduction des dépenses des collectivités ne passe pas seulement par un ralentissement des recrutements mais également par un recours de plus en plus fréquent aux formes d'emploi les plus flexibles (Biland, 2011, p. 44-50). Le désengagement de l'Etat et la contractualisation de l'emploi public contribuent ainsi à une dévaluation du capital scolaire sur le marché du travail local, qui va directement porter préjudice à la génération née dans les années 1980. Cette génération présente par ailleurs d'autres caractéristiques qui la rendent singulière. En premier lieu, des origines populaires plus marquées que leurs aînés ; la plupart sont en effet issus de ménages ouvriers autochtones. Ensuite, l'engagement dans une émigration étudiante, au sein des filières non-sélectives de l'université publique relatives aux humanités, y est plus fréquente. Enfin, l'expérience d'un retour contraint dans l'espace d'origine, en raison de leur élimination du système scolaire et/ou du marché du travail fortement concurrentiel des grandes agglomérations, y est abondamment partagée. Pour toutes ces raisons, cette génération est composée en majorité de femmes qui, parmi les classes populaires rurales ou originaires des villes petites et moyennes, s'engagent plus fréquemment dans une émigration étudiante que les hommes, mais sont aussi davantage réassignées à leur espace d'origine à l'issue de celleci $^{9}$.

Ce retour à Lergnes participe à contrarier leurs aspirations d'ascension sociale fondée sur l'accumulation de capital culturel ainsi que sur le désir de s'établir dans une grande agglomération. Les manifestations publiques de la petite bourgeoisie culturelle lergnoise ainsi que les sociabilités qui leur sont périphériques vont alors se présenter pour eux comme une scène d'actualisation et de reconnaissance du capital culturel rapporté de l'expérience de l'émigration étudiante, en dehors d'un emploi qui n'en offre que rarement la possibilité. Comme l'avaient fait leurs aînés dans les années 1980, ces jeunes étudiants de retour vont ainsi largement s'investir dans des activités militantes collectives (sous la forme associative) ainsi que dans des projets artistiques personnels, sans toutefois que s'offrent à eux la perspective d'une reconversion militante heureuse. Ainsi, cette deuxième génération n'apparaît pas seulement plus fragilisée sur le terrain de l'emploi, elle l'est tout autant sur la scène associative, au sein de laquelle les initiatives qu'elle porte ne sont pas soutenues par les partenaires publics et n'offrent pas des profits semblables à ceux obtenus vingt à trente ans plus tôt par leurs aînés. Au total, si le processus de fragilisation de la petite bourgeoisie culturelle s'exerce bien sur l'ensemble du groupe, les membres de la deuxième génération, composée en majorité de jeunes femmes d'origine modeste, sont plus directement concernés. Cet aspect n'est pas anodin ; c'est précisément parmi ces étudiants de retour aux aspirations sociales contrariées que vont s'observer les manifestations les plus aiguës du mépris de classe, que celles-ci soient adressées aux « cas soc' » ou aux «beaufs ».

10,1\% pour les personnes nées dans les années 1960, 21,2 \% pour celles nées dans les années 1970, et 58,2\% pour celles nées dans les années 1980 .

${ }^{9}$ Pour une analyse des processus d'assignation spatiale des jeunes femmes de classes populaires originaires des espaces ruraux et des villes petites et moyennes, voir Elie Guéraut (2018, p. 208-217). 


\section{Une légitimité contestée « par la droite ». Crise du socialisme municipal et recomposition de la scène associative locale}

\section{Mars 2014 : quand les socialistes perdent les élections municipales}

Si la fragilisation de la petite bourgeoisie culturelle procède de tensions structurelles qui dépassent l'espace local, la crise du socialisme municipal va contribuer à en redoubler les effets. Le 30 mars 2014, la liste d'union de la gauche, formée entre le PS, le PCF (Parti Communiste Français) et EELV (Europe Ecologie-Les Verts), perd au deuxième tour des élections municipales face à la coalition d'une liste « sans étiquette », UMP et UDI, élue sur la promesse d'une réduction de la fiscalité locale au prix d'une "politique globale de réduction des coûts $»^{10}$. Cette défaite des socialistes, la première depuis 1965 , occasionne en premier lieu une transformation radicale de la composition sociale du Conseil municipal. Jamais les chefs d'entreprise (4/39), les cadres du privé (9/39) et les professions libérales $(5 / 39)$ n'y avaient été autant représentés, tandis que le nombre de salariés directs ou indirects de la main gauche de l'Etat est au plus bas depuis 1983 (on en compte alors 12, contre 20 en 1989, 18 en 1995, 14 en 2001 et 21 en 2008). Ce déclin du socialisme municipal n'est bien sûr pas propre à Lergnes. Comme le montre par exemple Luc Rouban (2015), les élections municipales de 2014 se caractérisent par un net recul du PS et du PCF au profit de l'UMP, de l'UDI et de listes « divers droite », ainsi que par une transformation du profil sociologique des maires élus (on retrouve parmi eux bien davantage de chefs d'entreprise et de professions libérales qu'en 2008, tandis que la part des cadres salariés diminue). Le politiste ajoute que les questions de déclin des centres-villes et de périurbanisation ont souvent été au cœur des campagnes qui ont donné lieu à une victoire de la droite. De fait, le cas de Lergnes n'a rien de singulier mais rend compte avec exemplarité des transformations à l'œuvre dans l'ensemble des villes françaises, et plus particulièrement dans celles sujettes au déclin urbain.

Dès leur arrivé au pouvoir, ces nouveaux élus décident une série de mesures que la petite bourgeoisie culturelle lergnoise reçoit avec hostilité. D'abord, la délégation aux «affaires culturelles » est accordée à une allochtone arrivée à Lergnes en 2007, directrice d'une association visant à "l'insertion par l'activité économique », de ce fait largement en dehors des relations d'interconnaissance du groupe. La direction des «affaires culturelles » est ensuite supprimée de l'organigramme de la collectivité, remplacée par celle des «projets associatifs et événementiels » sous la tutelle de l'adjoint à « la Communication, au Tourisme aux Relations Extérieures » tout juste élu, un chef d'entreprise lergnois propriétaire de salles de sports. Par ailleurs, les grandes manifestations culturelles soutenues par la municipalité, à l'image d'une «nuit électro » où se produisent tous les 21 juin devant la mairie des disquesjockeys des boîtes de nuit de la région, ou encore d'un spectacle son et lumière relatant la vie d'une Sainte décédée à Lergnes, proposent pour la plupart des biens symboliques diamétralement opposés à ceux produits et/ou valorisés par la petite bourgeoisie culturelle lergnoise. Enfin, la réduction des dépenses de la collectivité atteint une ampleur jusqu'alors inédite, 1,6 millions d'euros en 2016 (-14\% entre 2015 et 2016), dont $388000 €$ sont

\footnotetext{
${ }^{10}$ Extrait du programme de la liste « sans étiquette ».
} 
ponctionnés sur les subventions aux associations ${ }^{11}$. Sont d'abord ciblées celles qui bénéficiaient jusqu'alors de ce que le nouveau maire appelle le «systématisme », «qui donnait à certains et pas à d'autres $»^{12}$. Sont ainsi concernées les associations culturelles créées et/ou contrôlées par des membres de la petite bourgeoisie culturelle, dont certaines perdent l'intégralité de leur subvention municipale, comme c'est le cas de celle proposant des ateliers d'arts plastiques aux enfants dirigée par une ancienne élue municipale socialiste. Une grande réorganisation des services est ensuite entreprise dans l'objectif de réaliser une économie de $236000 €^{13}$ sur la masse salariale. Celle-ci se traduit par un plan de départ volontaire à la retraite et le non-renouvellement d'une partie des vacataires et des contractuels, dont de nombreux membres de la deuxième génération de la petite bourgeoisie culturelle.

Cette nouvelle majorité municipale vient ainsi relayer plus durement une politique nationale de réduction des dépenses publiques mais aussi remettre en cause les « ressources clientélaires » (Mattina, 2004) dont pouvait bénéficier la petite bourgeoisie culturelle, notamment en organisant une réaffectation des ressources de la collectivité vers des associations, des entreprises et des particuliers liés au pôle économique de l'espace local. Ce faisant, la fragilisation dont elle fait l'objet va soudainement être associée à un groupe social incarné dans l'espace local : la bourgeoisie économique. Comme son homologue du pôle culturel, celle-ci constitue un milieu d'interconnaissance hiérarchisé, qu'il m'a été possible d'approcher par le biais de ses manifestations publiques et par les associations qui le composent : Lion's Club, Rotary Club, BNI (Business Network International), associations de commerçants, espaces d'entre-soi où se côtoient chefs d'entreprise, cadres du privé, commerçants et artisans, ainsi que des élus des collectivités (dont plusieurs accèdent au Conseil municipal en 2014) et des instances de représentations professionnelles.

\section{Disqualifier les « jeunesses économiques »}

La JCEL (Jeune Chambre Economique de Lergnes), dont j'ai été membre de 2014 à 2015 dans le cadre de mon enquête ethnographique, y occupe une position dominée, ce qui s'explique à la fois par son implantation récente (2011) et par les origines sociales modestes de nombre de ses membres, dont aucun n'est directement issu de la (petite) bourgeoisie économique locale. Soucieux de se faire pleinement intégrer à leur groupe de référence et encouragés par les élus municipaux (qui mettent à disposition de la JCEL un local dans le centre-ville au début de l'année 2015), les membres de l'association sont, au moment où je les rencontre, particulièrement actifs. Ils décident ainsi d'investir le domaine de la création et de la diffusion de biens symboliques, encore partiellement sous contrôle de la petite bourgeoisie culturelle lergnoise, en organisant un concours de création d'œuvres d'art. Le projet est porté par celui qui deviendra président de l'association l'année suivante, Thomas ${ }^{14}$, un lergnois de 33 ans, fils d'un ouvrier qualifié et d'une femme au foyer. Titulaire d'un BEP «métiers de la restauration et de l'hôtellerie », il est alors cuisinier dans un restaurant d'entreprise. Il n'a pas

\footnotetext{
${ }^{11}$ Idem.

${ }^{12}$ Maire de Lergnes, réunion publique du 16/09/2015.

${ }^{13}$ Compte-rendu du Conseil municipal du mois de décembre 2015.

${ }^{14}$ Les noms ont été changés.
} 
connu l'expérience de l'émigration étudiante et ne dispose pas des ressources culturelles qu'elle peut délivrer. Dès lors, ses aspirations à rejoindre la petite bourgeoisie économique se trouvent contrariées par la modestie des capitaux hérités et/ou acquis au cours de sa trajectoire, malgré cet engagement intense au sein de cette association (environ une vingtaine d'heures de travail bénévole par semaine), qui lui donne la possibilité de côtoyer la bourgeoisie économique lergnoise. J'assiste à plusieurs réunions de préparation de l'évènement où $\mathrm{j}$ 'apprends que les difficultés rencontrées sont nombreuses, notamment pour référencer les artistes locaux susceptibles d'y participer, constituer un jury de «professionnels » (le projet sera d'ailleurs avorté), ou encore trouver des lieux d'exposition appropriés. Au-delà de la distance au capital culturel, qui sera bientôt dénoncé avec force par les membres de la petite bourgeoisie culturelle afin de disqualifier la manifestation, c'est donc aussi un défaut de capital social efficient au sein du pôle culturel lergnois qui porte ici préjudice à Thomas.

A un mois du vernissage, les membres de la JCEL parcourent la ville, ses lieux de sociabilité et ses commerces, afin de distribuer les affiches annonçant la manifestation. C'est à cette occasion que Thomas entre dans le local d'une association culturelle, où je me trouve justement ce mercredi après-midi du mois de novembre 2014. Cette association n'est pas de celles créées dans les années 1980 par la première génération de la petite bourgeoisie culturelle lergnoise, mais de constitution plus récente (2013). Elle est au moment des faits très fréquentée (elle compte 1400 membres en 2014), ce qui s'explique par le grand nombre et la variété des manifestations proposées dans son local (concerts des groupes de rock locaux mais aussi de musiciens allochtones officiant dans des genres plus avant-gardistes, expositions d'art contemporain, conférences publiques, etc.). Lorsque Thomas entre dans le local de l'association, sans se douter du mépris dont il fera l'objet, on ne compte qu'une dizaine de personnes présentes, essentiellement des habitués, étonnés par l'arrivée du jeune homme. Chemise blanche rentrée dans un jeans neuf coupé près du corps, montre en métal au poignet, chaussures en cuir noires pointues et cheveux peignés en brosse, son apparence, qui fera a posteriori l'objet de moqueries, détonne face à celle des bénévoles de l'association, vêtus de leurs habituels baskets, jeans larges et sweats à capuche à l'effigie de groupes rock ou de leurs pullovers usés.

Thomas se dirige vers la personne la plus proche de l'entrée. Il s'agit d'Alice, une jeune lergnoise de 29 ans. Ses origines modestes (son père est commercial itinérant de produits de plomberie et sa mère aide à domicile), son expérience de l'émigration étudiante (elle obtient le diplôme de l'école des Beaux-arts de Lyon) et son retour à Lergnes sous contrainte (elle se sépare de son compagnon et ne trouve pas d'emploi à Lyon), font d'elle un cas emblématique des membres de la deuxième génération de la petite bourgeoisie culturelle lergnoise. Elle exerce, depuis ce retour, le métier de professeure documentaliste contractuelle, au gré de la vacance des postes, s'exposant parfois à de longues périodes d'inactivité. Présidente de l'association, elle gère depuis ses débuts l'espace dédié aux expositions. Thomas s'avance donc vers elle et lui présente à grands traits cette manifestation qu'il organise avec la JCEL. Pendant les quelques minutes que dure la présentation, Alice ne dit mot et n'accorde pas un regard à Thomas, qui, en réaction à cette indifférence manifeste, perd ses moyens. Le malaise ressenti par le jeune homme s'apprécie alors directement sur son 
corps (ses joues rougissent) mais aussi à travers des manières d'élocution qui viennent ici rappeler ses origines populaires et rurales et qu'il parvient habituellement à contenir en public (bafouillages, fautes de grammaire, léger accent régional). Cette présentation achevée, et devant l'indifférence d'Alice qui n'a toujours pas relevé les yeux, Thomas décide de laisser une affiche sur la table devant laquelle la jeune femme est installée et fuit littéralement la scène, ne se retournant même pas vers le comptoir du bar où je suis installé avec les autres membres de l'association qui observent la scène en silence ${ }^{15}$. L'affiche en question, après avoir suscité rires et plaisanteries, est finalement saisie par Alice et littéralement mise en morceaux.

Quelques semaines plus tard, la jeune femme revient à l'occasion d'un entretien sur cette confrontation avec ce représentant de ceux qu'elle a depuis pris l'habitude de surnommer «les jeunesses économiques », en référence aux «jeunesses hitlériennes ${ }^{16}$, expression qui, à force de « commérage réprobateur » (Elias, 1985, p. 26), s'est diffusée dans l'entre-soi de la petite bourgeoisie culturelle lergnoise. Dans les extraits qui suivent, elle s'exprime avec Stéphane ${ }^{17}$ qui s'est joint à l'entretien qui se déroule dans le local de l'association.

Alice : - « Je me rappelle du type qui est venu me filer des affiches et des invitations [...]. Il nous a dit que c'était la Jeune Chambre Économique et il nous a demandé si on était intéressés, j'ai fait [elle fait une grimace] Je l'ai laissé parler [...] et j'ai tout foutu à la poubelle. [...] Je comprenais pas ce qu'il foutait là, dans ce truc pour l'art alors qu'il y comprenait visiblement rien. [...] Je comprenais pas le lien : «Qu'"est-ce que tu fous là ? Qu'est-ce que t'es en train de me dire ? ». Donc je ne t'écoute pas, ça ne me concerne pas. ».

[Je leur montre ensuite une copie de l'affiche (figure 1) de la manifestation présente sur mon ordinateur]

Stéphane [en regardant l'affiche] : «Pour moi c'est pas de la culture [...]. Nan puis... Nan moi déjà ça [il montre le titre de la manifestation], voilà, déjà ça c'est hideux. Mais n'empêche que c'est symptomatique d'une certaine vision de la culture voilà quoi. C'est... [titre de la manifestation], non quoi !».

[Rires de Stéphane et d'Alice - je leur montre l'œuvre gagnante]

$\mathrm{S}:$ «Non mais... c'est horrible. [...]. J'appelle ça de la culture IKEA : c'est pas un tableau, c'est pas de la peinture, c'est euh... c'est un papier peint quoi. ».

[Il revient sur l'affiche]

$\mathrm{S}$ : «Et puis ça c'est... un fond d'écran de téléphone portable quoi. Puis le texte quoi ! Déjà un titre avec des apostrophes... ».

Ces propos rendent compte du profond dégoût exprimé par Alice et Stéphane à la vision des tableaux et de l'affiche de la manifestation, que ceux-ci refusent d'ailleurs de considérer comme « de la culture ». Le tableau de lauréat est par exemple jugé emblématique

\footnotetext{
15 Ainsi, Thomas n'a sur le moment même pas remarqué ma présence dans le local. J'aurais néanmoins l'occasion de discuter de la scène avec lui après la tenue de la manifestation. Celui-ci me dira alors ne pas comprendre la raison du mépris manifesté par Alice à son égard et, en conséquence, que la jeune femme serait « complétement folle».

${ }^{16}$ L'utilisation de cette expression montre que la distance sociale qui sépare ces deux jeunesses recouvre pour Alice une dimension éthique et politique forte. Elle vise aussi à disqualifier l'incursion de la JCEL dans le domaine culturel en faisant valoir leur proximité supposée aux idées de la droite et de l'extrême-droite, qu'elle juge incompatibles avec l'exercice d'activités de production ou de diffusion de biens culturels.

${ }^{17}$ Stéphane, âgé de 40 ans, est né à proximité de Lergnes d'un père mécanicien et d'une mère secrétaire de mairie, ancien salarié d'une association culturelle dont il a été licencié en 2008 (il enchaine depuis divers petits boulots en CDD auprès des collectivités de la région, il est alors agent d'accueil touristique dans une commune proche).
} 
d'une culture de masse («IKEA»), inscrite dans des objets de consommation courants, et dénuée de toute valeur symbolique positive. La photographie de la remise des prix que je leur montre provoque également l'indignation. Le «style associatif » (Bozon, 1982, p. 1970) que l'on y observe (le port du costume par exemple, et plus largement la mise en scène sophistiquée que l'on devine - figure 2) témoigne d'un haut degré de formalisme que l'on ne retrouve effectivement pas dans des manifestations de la petite bourgeoisie culturelle, qui, elles, donnent à voir une distance maîtrisée aux normes de présentation de soi des classes supérieures. Ainsi, ce que moquent ici Stéphane et Alice, ce n'est pas seulement la distance au capital culturel des membres de la JCEL que l'on devine à travers les clichés de la manifestation ou à examiner l'ethos de Thomas, c'est aussi une modalité particulière de «bonne volonté culturelle» (Bourdieu, 1979, p. 367), essentiellement portée sur un style de vie bourgeois et conservateur ${ }^{18}$ plutôt que sur des pratiques ou des valeurs directement issues des univers scolaires ${ }^{19}$.

Ces jugements procèdent ainsi de dispositions éthiques, esthétiques, ainsi que de « morales de classe » (Caveng et al., 2018) socialement situées, et répondent à une logique de luttes symbolique pour imposer une vision légitime de la culture. A ce titre, la petite bourgeoisie culturelle n'a pas le monopole de l'expression du dégout de l'autre. Par exemple, l'ascétisme matériel des «fonctionnaires », des « cultureux» ou encore des «profs », qui ne les conduirait qu'à porter des vêtements usagés et démodés, à acheter les lunettes « les moins chères et remboursées par la mutuelle $\gg^{20}$, ou encore à ne conduire que des voitures en mauvais état, est très régulièrement moqué au sein des espaces d'entre-soi de la (petite) bourgeoisie économique. Mais dans la situation qui vient d'être décrite, ce dégoût de l'autre s'exprime directement aux personnes concernées, dans une interaction en face-à-face, se faisant alors mépris de classe. Il faut pour le comprendre rappeler que cette confrontation exceptionnelle entre Thomas et Alice s'inscrit dans un contexte de contestation de la légitimité de la petite bourgeoisie culturelle lergnoise. Au moment des faits, le premier, déjà désigné futur président de la JCEL, est parvenu à s'attirer les faveurs des élus municipaux, qui subventionnent la manifestation culturelle. Ce soutien de la municipalité aux «jeunesses économiques » est alors vivement critiqué par les membres de la petite bourgeoisie culturelle. Il est jugé encore plus sévèrement par Alice, qui voit dans un même temps le local de l'association qu'elle préside être menacé de fermeture par la municipalité, faute de la présence d'une issue de secours (la fermeture interviendra effectivement quelques mois plus tard). Impuissante à sauvegarder l'existence de son association et témoin privilégiée de l'incursion réussie de la JCEL sur le terrain de la diffusion d'œuvres d'art, Alice tente alors de contester la légitimité de cette présence sur la scène associative en se faisant le relais d'un ordre symbolique légitime qu'elle maîtrise mieux que son adversaire. Le registre du mépris de

\footnotetext{
${ }^{18} \mathrm{Au}$ sens que lui accordent Norbert Elias et Pierre Bourdieu, c'est-à-dire relatif à l'ancienneté et à la stabilité de la consécration des pratiques et goûts concernés.

${ }^{19}$ Cette modalité singulière de «bonne volonté culturelle » rapproche ainsi les membres de la Jeune Chambre Economique des employés des hôtels de luxe enquêtés par Amélie Beaumont (2017), qui, eux aussi, tendent à diriger leur attrait pour la culture légitime vers ses manifestations les plus évidemment bourgeoises et conservatrices : la consommation de produits de luxe (dîners et déjeuners dans les « grandes tables », port de vêtements et d'accessoires des marques des couturiers, etc.).

${ }^{20}$ Cette remarque sur la consommation de lunettes des fonctionnaires a été formulée à plusieurs reprises devant moi par un opticien franchisé, membre de la JCEL.
} 
classe participe alors à renforcer le pouvoir symbolique dont elle use afin d'imposer à son adversaire le sentiment de sa propre illégitimité, qui se matérialise chez lui sous la forme « d'émotions corporelles — honte, humiliation, timidité, anxiété, culpabilité — (...) d'autant plus douloureuses qu'elles se traduisent dans des manifestations visibles, comme le rougissement, l'embarras verbal, la maladresse, le tremblement, la colère ou la rage impuissante » (Bourdieu, 1997, p. 203). Ce faisant, l'objectif est autant de disqualifier cette concurrence nouvelle que d'asseoir sa position au sein de la petite bourgeoisie culturelle, en faisant valoir la distance qui la sépare désormais de ses origines populaires.

Figure 1 Affiche annonçant l'exposition organisée par la Jeune Chambre Economique de Lergnes « Cré ART'58 »

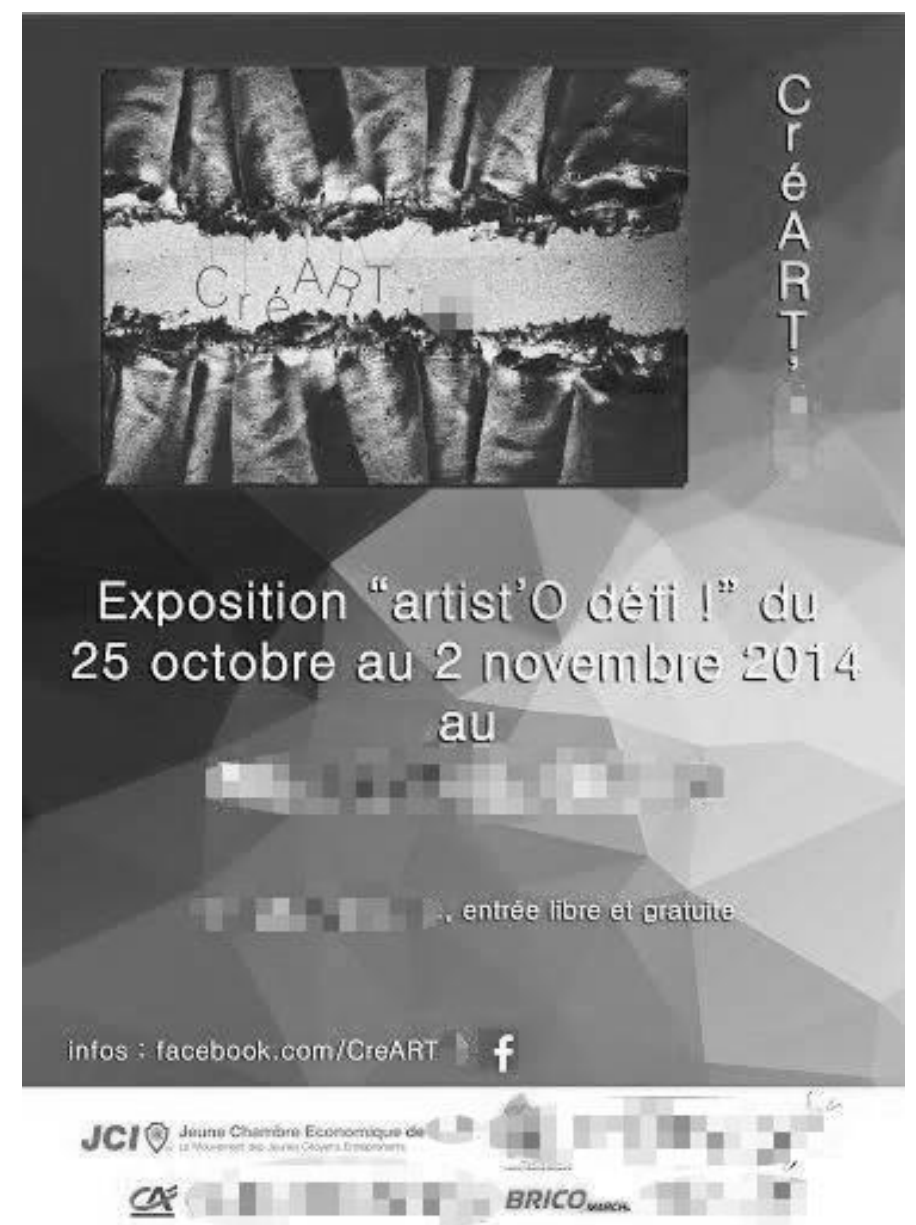

Figure 2 : Remise des prix aux lauréats à l'occasion de la fin de l'exposition organisée par la JCEL 


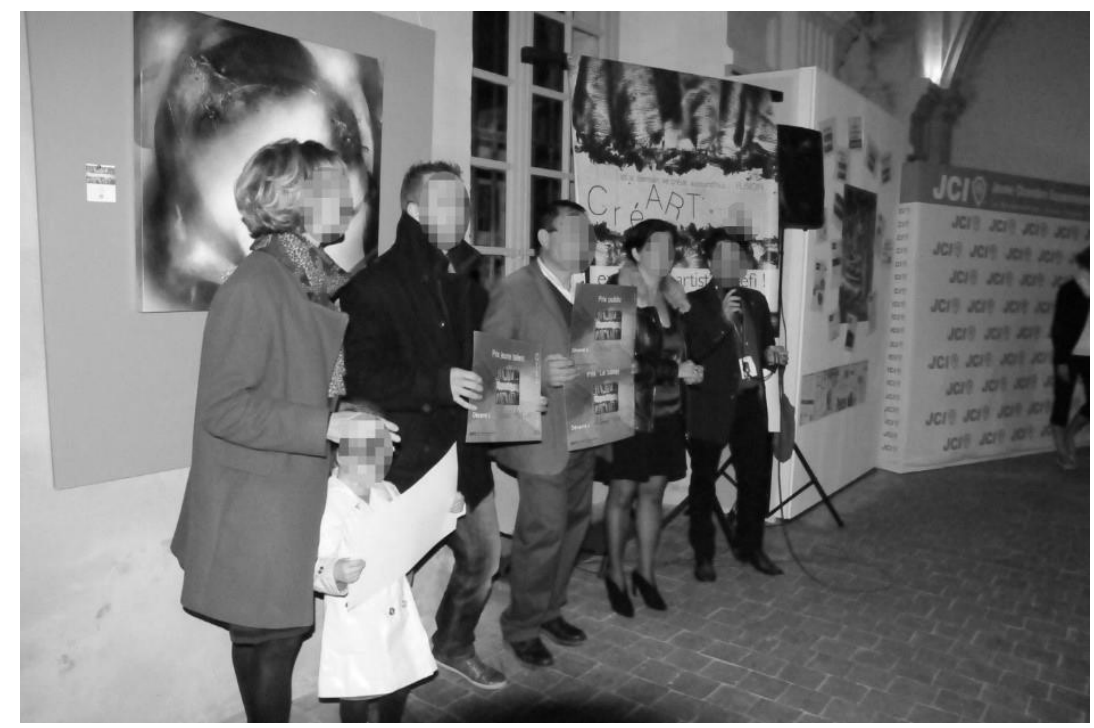

\section{Une légitimité contestée «par le bas». Paupérisation et décroissance des quartiers centraux}

\section{Quand le déclin de la ville gagne les quartiers centraux}

La petite bourgeoisie économique n'est cependant pas le seul groupe social de l'espace local à incarner, aux yeux des membres de la petite bourgeoisie culturelle, la fragilisation dont ils font l'objet. La difficulté croissante qu'ils rencontrent à conformer leur espace résidentiel à leur style de vie, en raison de la dynamique de déclin qui s'y observe, est, dans les discours, explicitement reliée à la présence toujours plus visible d'un voisinage jugé indésirable, les «cas soc' ${ }^{21}$. Les nombreuses enquêtes portant sur les dynamiques de gentrification, en France ou dans d'autres pays, ont largement mis en évidence la tendance des fractions culturelles des classes moyennes et supérieures à aménager l'espace public dans le but de le conformer à leur style de vie. Ce «travail de gentrification» (Bidou-Zachariasen et Poltorak, 2008) mené depuis les années 1980 par la petite bourgeoisie culturelle lergnoise se traduit par une transformation de l'espace public : prise de contrôle de cafés et de commerces, ouverture de nombreux locaux associatifs où s'organisent concerts et expositions d'art, etc. Mais c'est peut-être plus encore par l'appropriation ordinaire de l'espace public que ce phénomène s'apprécie : fêtes de quartiers où se produisent des groupes de rock, manifestations culturelles sur les places du centre-ville, repas de voisins dans les cours des immeubles où l'interconnaissance est générale, etc. Au moment de l'enquête, ce travail de gentrification sur l'espace résidentiel est mis à mal par plusieurs facteurs. Comme on l'a vu, la nouvelle municipalité se désengage de nombreuses associations culturelles, et dans quelques cas des procédures sont même menées pour faire fermer certains locaux sur arrêté municipal ou en réduire la fréquentation, justifiant ces procédures par des raisons de sécurité. Ensuite, la petite

\footnotetext{
${ }^{21}$ La mobilisation de cette catégorie du sens commun pour qualifier cette altérité populaire indésirable montre que celle-ci n'a pas seulement court au sein des classes populaires, où elle permet d'appuyer les stratégies de distinction des fractions les plus établies, comme le montre par exemple Raphaël Challier dans ce numéro.
} 
bourgeoisie culturelle, qui a toujours privilégié une localisation dans les quartiers centraux ${ }^{22}$, se trouve aujourd'hui davantage contrainte de partager cet espace avec des classes populaires précarisées, en partie originaires des communes ouvrières et rurales alentours ${ }^{23}$.

Le phénomène s'apprécie à l'examen des recompositions de la structure sociale de la commune de Lergnes et des trois IRIS centraux entre 1990 et 2013 (tableau 1). Par exemple, la part de cadres et professions intellectuelles supérieures décroit légèrement sur la période 2006-2013 dans deux des trois IRIS de centre-ville (de 1 à $2 \%$ ), tandis qu'elle grimpe de $2 \%$ à l'échelle communale. A contrario, la part d'ouvriers est en progression dans deux des trois IRIS du centre-ville (de 4 et $6 \%$ ) sur la même période alors qu'elle est en diminution de $2 \%$ à l'échelle communale. Mais la progression la plus spectaculaire concerne le taux de chômage ${ }^{24}$. Tandis qu'en 1990 le phénomène est sous-représenté dans les quartiers centraux, il gagne en importance dans les décennies qui suivent, jusqu'à dépasser à partir de 2006 la moyenne communale dans deux des trois IRIS de centre-ville. En 2013, ce taux va même jusqu'à atteindre $25 \%$ dans un de ces IRIS, contre $21 \%$ à Lergnes.

Tableau 1 : Décroissance démographique, vacance immobilière et recomposition de la structure sociale des quartiers centraux de Lergnes de 1990 à 2013

\begin{tabular}{|c|c|c|c|c|}
\hline & 1990 & 1999 & 2006 & 2013 \\
\hline \multicolumn{5}{|c|}{ Part logements vacants (en \%) } \\
\hline Iris centre-ville historique & 15 & 13 & 17 & 21 \\
\hline Iris centre-ville résidentiel & 11 & 12 & 10 & 16 \\
\hline Iris centre-ville commerçant & 13 & 20 & 17 & 28 \\
\hline Commune de Lergnes & 8 & 10 & 12 & 17 \\
\hline \multicolumn{5}{|c|}{ Nombre d'actifs de 15 à 64 ans (indice base 100) } \\
\hline Iris centre-ville historique & $1263(100)$ & $1282(102)$ & $1272(101)$ & $1200(95)$ \\
\hline Iris centre-ville résidentiel & $1133(100)$ & $1234(109)$ & $1210(107)$ & $1076(95)$ \\
\hline Iris centre-ville commerçant & $1382(100)$ & $1371(99)$ & $1423(103)$ & $1287(93)$ \\
\hline Commune de Lergnes & $18945(100)$ & $18254(96)$ & $16500(87)$ & $14545(77)$ \\
\hline \multicolumn{5}{|c|}{ Part des cadres et professions intellectuelles supérieures (en \%) parmi les actifs de 15 à 64 ans } \\
\hline Iris centre-ville historique & 12 & 16 & 21 & 19 \\
\hline Iris centre-ville résidentiel & 20 & 20 & 19 & 18 \\
\hline Iris centre-ville commerçant & 13 & 16 & 18 & 21 \\
\hline Commune de Lergnes & 10 & 11 & 13 & 15 \\
\hline \multicolumn{5}{|c|}{ Part des professions intermédiaires (en \%) parmi les actifs de 15 à 64 ans } \\
\hline Iris centre-ville historique & 24 & 28 & 24 & 28 \\
\hline Iris centre-ville résidentiel & 16 & 27 & 29 & 29 \\
\hline Iris centre-ville commerçant & 18 & 27 & 28 & 25 \\
\hline Commune de Lergnes & 19 & 22 & 22 & 24 \\
\hline Part des en & yés $($ en \%) pa & i les actifs de & 5 à 64 ans & \\
\hline
\end{tabular}

\footnotetext{
${ }^{22} \mathrm{Au}$ contraire des cadres et des professions intermédiaires de l'agglomération lergnoise pris dans leur globalité qui se dirigent depuis les années 1990 vers des communes résidentielles limitrophes.

${ }^{23}$ Ainsi, 15,3\% des nouveaux arrivants résidaient dans une autre commune de l'unité urbaine et 48,6 \% dans une autre commune du département (sources : Insee, RP 2013).

${ }^{24} \mathrm{Au}$ sens du recensement.
} 


\begin{tabular}{|ccccc}
\hline Iris centre-ville historique & 27 & 29 & 30 & 26 \\
\hline Iris centre-ville résidentiel & 29 & 30 & 33 & 28 \\
\hline Iris centre-ville commerçant & 29 & 30 & 29 & 28 \\
\hline Commune de Lergnes & 32 & 34 & 33 & 32 \\
\hline Part des ouvriers (en \%) parmi les actifs de $\mathbf{1 5}$ à $\mathbf{6 4}$ ans & \\
\hline Iris centre-ville historique & 29 & 20 & 25 & 31 \\
\hline Iris centre-ville résidentiel & 20 & 15 & 15 & 19 \\
\hline Iris centre-ville commerçant & 22 & 17 & 27 & 24 \\
\hline Commune de Lergnes & 33 & 29 & 27 & 25 \\
\hline Part des chômeurs (en \%) parmi les actifs de $\mathbf{1 5}$ à $\mathbf{6 4}$ ans & 15 & 25 \\
\hline Iris centre-ville historique & 13 & 14 & 15 & 21 \\
\hline Iris centre-ville résidentiel & 9 & 11 & 12 & 19 \\
\hline Iris centre-ville commerçant & 13 & 15 & 14 & 21 \\
\hline Commune de Lergnes & 14 & 16 & 15 \\
\hline
\end{tabular}

Source : Recensements de la population 1990, 1999, 2006 et 2013 : tableaux profils, INSEE (producteur), ADISP-CMH (diffuseur)

Indication de lecture : Après 1999, le recensement s'appuie sur un échantillon aléatoire de logements. L'ensemble des intervalles de confiance a donc été calculé pour les variables renseignant les taux des différentes PCS dans la population active. Afin de ne pas alourdir la lecture de ce tableau, ces derniers n'ont cependant pas été indiqués. L'ampleur des intervalles variant ici, selon les cas, entre 2 et 4 points, les comparaisons des différents taux sont d'autant plus interprétables que l'écart qui les sépare est grand et qu'il dépasse 4 points.

Cette présence accrue de ménages modestes s'explique par un effritement des frontières économiques qui pouvaient jusqu'alors les tenir à distance du centre-ville. En effet, le prix de l'immobilier à Lergnes ne cesse de baisser depuis $2006^{25}$, en lien avec l'augmentation de la vacance résidentielle ${ }^{26}$, en particulier au sein des quartiers de centreville. La décroissance et la paupérisation de Lergnes s'accompagnent ainsi d'une mutation du système de ségrégation urbaine. Du «modèle de ségrégation centre-périphérie » qui prévalait en 1990, s'est substitué dans la période contemporaine celui de «ségrégation centrepériphérie inversé »(Cusin, 2016). Une telle transformation contribue à la fragilisation de la petite bourgeoisie culturelle lergnoise dans la mesure où elle implique une perte de contrôle sur son espace résidentiel. En effet, la transformation de la morphologie sociale du centreville précipite la fermeture des commerces que fréquentent ses membres (le taux de vacance commerciale atteint plus de $15 \%$ en $2015^{27}$ ) et engendre l'arrivée de nouvelles boutiques (de restauration rapide et de vêtements bon marché pour l'essentiel) en rupture avec leur style de vie. De même, les modalités d'occupation de l'espace public de ces nouveaux habitants discussions sur les places du centre-ville parfois jusque tard dans la nuit, activités mécaniques de rue, volume élevé de la musique qui sort des fenêtres ouvertes - fragilisent le travail

\footnotetext{
${ }^{25}$ Selon le site internet meilleursagents.com (consulté le 13 juin 2019) qui s'appuie sur les données Perval, le prix moyen du m2 à Lergnes, situé autour de $1000 €$ en 2019, aurait perdu $8 \%$ de sa valeur entre 2009 et 2019.

${ }^{26}$ A l'échelle de la commune, le taux de logement vacant passe de 7,7 \% en 1990 à 16,8 \% en 2013 (tableau 1).

27 Selon un rapport de l'Inspection générale des finances et du Conseil général de l'environnement et du développement durable sur le déclin des centres-villes.
} 
d'appropriation matérielle et symbolique que la petite bourgeoisie culturelle mène sur les quartiers centraux depuis les années 1980. Au contraire des dynamiques de gentrification observées dans certains quartiers des grandes agglomérations, celles à l'œuvre à Lergnes échappent ainsi au contrôle de la petite bourgeoisie culturelle, alors contrainte à une mixité sociale dont elle n'a pas défini les modalités. La présence des classes populaires précarisées au sein de leur espace résidentiel, désignées par l'expression de «cas soc' », est alors explicitement regrettée.

Le constat de leur progression se fait le plus souvent par le récit d'anecdotes venant faire la démonstration de leur distance à la culture légitime, si ce n'est parfois à la culture tout court. Les "cas soc'» seraient ainsi responsables des dépôts d'encombrants sauvages, des entorses au règlement sur le tri des déchets, ou encore de l'odeur d'urine constatée à proximité de certains cafés. La vive allure des véhicules en centre-ville et la consommation de stupéfiants et d'alcool dans l'espace public, comme d'autres comportements jugés à risque, sont également vivement dénoncés. Les critiques pointent plus généralement un manque de « civisme » : volume élevé de la musique qui sort des voitures ou des fenêtres d'appartements, absence de salutations entre voisins, vols et faits de petite délinquance, etc. L'image ainsi construite est largement façonnée par un «ethnocentrisme de classe » associant ces voisins indésirables à la «barbarie », à la «nature » et à l' « inculture » (Grignon et Passeron, 1989, p. 37). Toutes les fractions des classes populaires ne sont pas concernées par cette assignation à la « minorité du pire » (Elias, 1985), mais seulement celles qui, en raison de comportements jugés déviants supposés ou constatés, viennent contrarier le travail de gentrification mené par la petite bourgeoisie culturelle sur les quartiers centraux. Elle ne concerne ainsi pas les classes populaires qui conservent leur distance au centre-ville, en particulier celles constituées d'immigrés et de descendants d'immigrés originaires d'Afrique du Nord et de Turquie, concentrées dans les quartiers d'habitat social périphériques ${ }^{28}$. Les membres de la petite bourgeoisie culturelle ne manquent ainsi pas de distinguer une altérité populaire désirable d'une altérité populaire indésirable. L'assignation à la figure du « cas $\operatorname{soc}^{\text {' } » ~ e t ~ a ̀ ~ l ' e n s e m b l e ~}$ des attributs négatifs qu'elle charrie concerne en premier lieu les classes populaires nouvellement installées dans le centre-ville de Lergnes, qui, par leur présence, viennent signifier à la petite bourgeoisie culturelle lergnoise le déclin de leur espace résidentiel ainsi que la fragilisation qui gagne le groupe.

Figure 4 : Une boulangerie fermée dans une rue de centre-ville (avril 2017)

\footnotetext{
${ }^{28}$ Le taux d'immigrés reste en effet faible dans les IRIS de centre-ville (de 3 à $6 \%$ ), tandis qu'il est compris entre 15 et $30 \%$ dans les IRIS classés en ZUS (Zone Urbaine Sensible), et qu'il est de 8,7\% en France. Sources : Insee 2012, données consultées sur https://sig.ville.gouv.fr/ le 09/04/2018.
} 


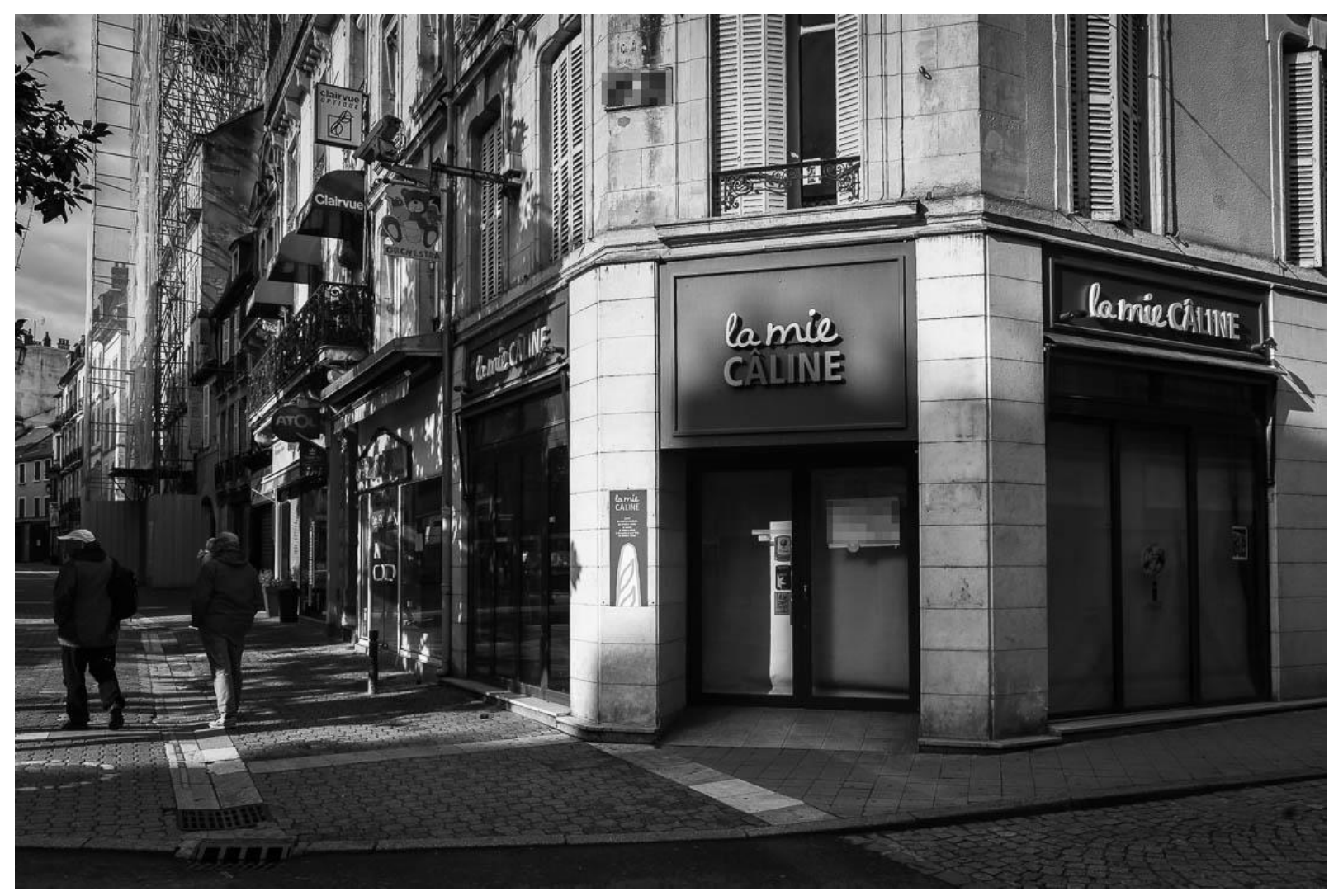

Photographie Aït Belkacem. Reproduite avec l'autorisation de l'auteur.

\section{«JE SUIS SURE QU'ILS VOTENT FN »}

Cette manifestation particulière de l'«ethnocentrisme de classe » se dévoile ici sans stratégie de dissimulation ou d'euphémisation, contrairement à ce qu'il est pourtant courant d'observer parmi les groupes sociaux politisés à gauche et positionnés dans les régions de l'espace social caractérisées par la prédominance du capital culturel. Cette contradiction entre les actes et les dispositions éthiques affichées (notamment au sein de la première génération de la petite bourgeoisie culturelle ${ }^{29}$ ), la « solidarité », la «tolérance », le « respect », la lutte contre «l'exclusion», est cependant dépassée du fait que soient prêtées à ces nouveaux habitants du centre-ville des opinions xénophobes. Cette caractéristique est une fois de plus produite par un travail intensif de commérage. Les jours suivant les élections, le constat récurrent du grand nombre de voix en faveur du FN dans les bureaux du centre-ville est commenté avec inquiétude, de même que les campagnes d'affichage que mènent les militants identitaires locaux (figure 5), ou encore l'ouverture de lieux de sociabilité soupçonnés d'être fréquentés par des sympathisants d'extrême-droite ${ }^{30}$. L'association entre la présence des « cas soc' » et les stigmates de la progression des idées d'extrême-droite dans l'espace local est alors énoncée sur le mode de l'évidence ; «je suis sûre qu'ils votent FN » me dira par

\footnotetext{
${ }^{29}$ A l'instar de la génération des «pionniers » dépeinte par Anaïs Collet (2015), la politisation de la première génération et les pratiques militantes qui en découlent sont bien plus importantes que celles des générations suivantes.

${ }^{30}$ Pour une étude plus complète du phénomène à partir de l'analyse des réactions suscitées par l'ouverture d'un café-concert suspecté d'être tenu et fréquenté par des personnes politisées à l'extrême-droite, Elie Guéraut (2017).
} 
exemple une cadre de la FPT de 54 ans $^{31}$, alors que je la croise dans la rue où nous habitons tous les deux et que sortent d'un immeuble trois jeunes hommes blancs aux cheveux très courts vêtus de survêtements, dont l'un d'eux a pris l'habitude de garer sa moto dans la cour de l'ancien hôtel particulier qu'elle habite, malgré son opposition.

Figure 5 : Des autocollants du mouvement politique « Génération identitaire » dans le centre-ville

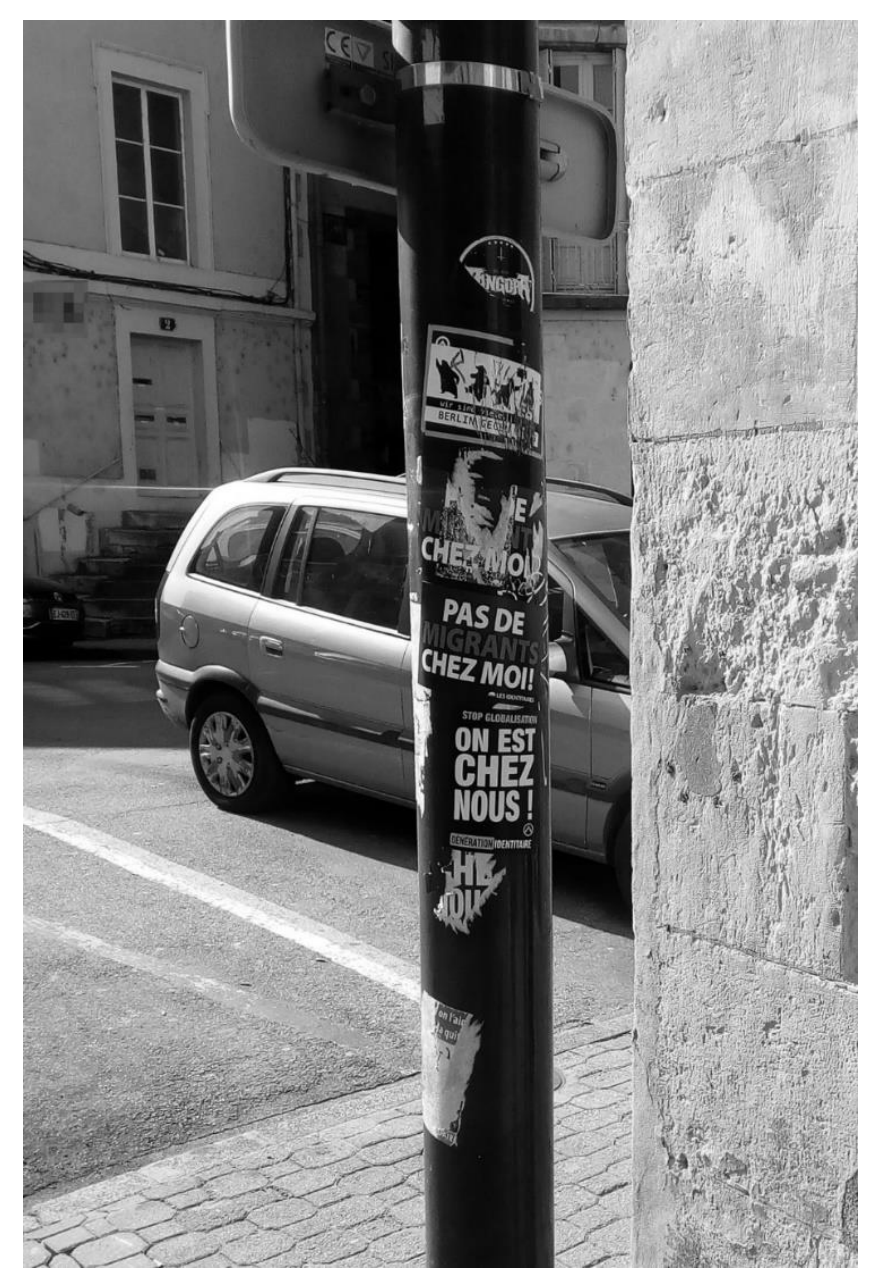

Les occasions d'interaction avec ces «cas soc'» suspectés de racisme restent cependant très limitées, et rares sont les conflits directs auxquels j'ai pu assister. Celui sur lequel je me propose de revenir tout de suite prend naissance sur un support numérique de sociabilité, la page Facebook officielle de la commune de Lergnes, suite à un message annonçant «l'accueil de huit familles syriennes » posté en septembre 2015. La publication est visionnée près de 20000 fois $^{32}$ et suscite environ 500 commentaires, dont la majorité vise à critiquer la décision («Où seront-ils logé ? Au frais de qui ? » dit par exemple un homme). Face à cette levée de boucliers, une trentaine de membres de la petite bourgeoisie culturelle investissent ce support de sociabilité et tentent de disqualifier les propos qui y sont tenus en faisant valoir l'autorité symbolique conférée par leur position sociale et leurs activités militantes dans l'espace local. C'est par exemple le cas de Fabien, 31 ans, aide-soignant au

\footnotetext{
${ }^{31}$ Mariée avec un des militants associatifs des années 1980 reconvertis en politique.

${ }^{32}$ Chiffre communiqué par le service communication de la commune.
} 
centre hospitalier de Lergnes, proche de la petite bourgeoisie culturelle en raison de ses engagements associatifs (au sein d'une association commercialisant un ordinateur « éthique et écologique ») et politiques (au sein du parti Nouvelle Donne). Il inscrit dans les commentaires qui suivent la conversation, donc visibles par tous, un long message dans lequel il s'adresse aux « racistes, xénophobes et autres polyhandicapés du smoothie [cerveau] » (figure 6). Celuici se présente comme une série de conseils ironiques à destination de ceux qui s'opposent à cette décision, afin de «ne plus passer pour un abruti auprès des gens cultivés ». L'utilisation de ce procédé sarcastique lui permet, en filigrane, d'attaquer leur légitimité à prendre la parole en public sur le principe de leur distance au capital culturel. Le premier conseil vise ainsi à moquer les difficultés constatées que rencontrent certains à s'exprimer dans un langage scolaire («Procure-toi un Bescherelle»). Les suivants concernent plus spécifiquement les pratiques culturelles supposées de ces « racistes »: Fabien les incite à expérimenter la lecture de livres (d'abord sérieux, sur l'histoire européenne du XXe siècle puis, plus méprisant, «T'choupi », «Petit ours brun » et Marc Levy) et à se détourner de certaines pratiques jugées abêtissantes («où les points de QI tombent plus vite que les minutes ») : regarder TF1, la chasse, les «meetings de Sarkozy » ou encore les « rassemblements de tuning ».

\title{
Figure 6 : Fabien attaque la légitimité d'internautes à prendre la parole
}

\author{
Amis racistes, xénophobes et autres polyhandicapés du \\ smoothie, \\ Etre con n'est pas une fatalité, il existe même quelques petits trucs pour t'en \\ sortir et ne plus passer pour un abruti auprès des gens cultivés : \\ 1) Tu aimes la France et tu veux la défendre ? C'est bien! Alors par pitié \\ respecte au moins sa langue ! Procure-toi un Bescherelle, et tes diatribes \\ haineuses sur Internet n'en seront que plus crédibles, et qui sait, léchées \\ d'un certain lyrisme?
}

2) Achète un livre d'Histoire (pas drôles genre Jean Roucas, celle avec un grand $\mathrm{H}$, niveau 3ème ça suffira) et attarde toi sur l'Italie, l'Allemagne et la France des années 1920-30 et 40. C'est poilant et tu relèveras peut-être même quelques similitudes avec certains discours actuels de gens « qui disent pas que des conneries ». Pas d'inquiétude, il y a aussi des images et ça t'évitera de commettre de gros impairs historiques.

3) Éteins la télévision ! Fuis particulièrement TF1, comme si ton découvert autorisé en dépendait, surtout aux environs de $13 \mathrm{~h}$ oủ les points de $\mathrm{Ql}$ tombent plus vite que les minutes.

4) Lis des livres. Au début ça peut irriter les yeux, mais une paire de lunettes suffira à remédier au problème. En cas de grosses difficultés, commence par des lectures simples : T'choupi, Petit Ours brun, L'âne Trotro, Marc Levy...

5) Apprends à identifier et éviter les situations susceptibles de te faire rechuter: la chasse, les réunions d’anciens combattants, les meetings de Sarkozy, les rassemblements de tuning, les repas de famille avec l'oncle qui a fait la guerre d'Algérie etc.

Ça ne sera pas simple, et peut-être même que par moment tu seras découragé et tenté de pousser un « Le Pen vite » ou * la France tu l'aimes ou tu la quittes $»$. Dans ce cas, pose-toi et respire un grand coup, tu seras pas moins con mais on aura la paix! J'aime Répondre - $321-7$ septembre, 09:53-Modifié

On compte également de nombreux échanges interpersonnels (qui restent cependant visibles par tous). Celui qui est reproduit dans la figure 7 met en scène Alice et son conjoint, technicien-son et administrateur d'un groupe de musique lergnois (sous le statut d'intermittent du spectacle) né dans la région en 1978, à deux internautes critiquant l'accueil des huit 
familles syriennes. Alors que l'un d'eux fait valoir sa «liberté d'expression », l'autorisant selon lui à faire publiquement part de son désaccord, Alice lui répond directement et feint l'incompréhension ( «j'ai pas compris »), avant de s'essayer à parodier les difficultés de celuici à s'exprimer dans un français scolaire (« jé par conpprit »). Une femme se mêle alors à la conversation et dénonce le mépris dont elle se dit victime (« arrêtez de nous prendre pour des cons »), avant de laisser planer la menace d'un vote FN (« Quand est ce qu'on vote bordel »). Le conjoint d'Alice maintient pourtant la stratégie mise en place par cette dernière en commettant volontairement plusieurs fautes d'orthographe sur les deux mots de sa courte intervention ( « au ailéxion ? »). Le lendemain, je rencontre le couple dans un café du centreville prisé de la petite bourgeoisie culturelle, tenu par un ancien élu socialiste. Installés en terrasse avec Stéphane, ils discutent de la polémique suscitée par cette annonce et s'accordent à trouver scandaleuses les réactions de ces internautes. Nous examinons ensuite tous les quatre ces commentaires; les fautes d'orthographe les plus grossières sont relevées à voix haute, suscitant l'amusement général. Le conjoint d'Alice consulte alors le profil Facebook d'une des internautes visées et nous montre, hilare, un montage de plusieurs clichés où l'on peut voir cette femme, son compagnon et leurs enfants. Plusieurs éléments de la photo font ensuite l'objet de moqueries : le polo de l'homme, rose à rayures bleu marine, la décoration de noël en arrière-plan jugée « cheap » (bon marché), ainsi que les postures et la corpulence des corps ${ }^{33}$ en représentation. Finalement, Stéphane met fin à cet exercice collectif de critique et conclut : « une belle bande de cas soc'».

Le racisme supposé ou constaté des nouveaux habitants du centre-ville et de ces internautes hostiles à l'accueil de familles de réfugiés politiques, réunis sous la même désignation de «cas soc'», permet ainsi de neutraliser les dispositions éthiques qui conduisent habituellement les fractions intellectuelles des classes moyennes et supérieures à euphémiser toute expression du mépris de classe. Malpropres, dangereux, incultes et irrationnels, voire même dénués de toute forme d'intelligence, les « cas soc' » sont ici perçus au prisme d'un ethnocentrisme de classe débridé, fonctionnant sur la «stigmatisation moralisante du racisme ordinaire » qui lui sert «d'occasion et d'alibi » (Grignon et al., 1991, p. 42).

\section{Figure 7 : Alice et son conjoint parodient les difficultés d'internautes à s'exprimer dans}

\section{un langage scolaire}

\footnotetext{
${ }^{33}$ Pour une analyse de la charge moralisante que comporte la critique du surpoids et de l'obésité, par ailleurs plus fréquents parmi les classes populaires, voir Dieter Vandebroeck (2015).
} 


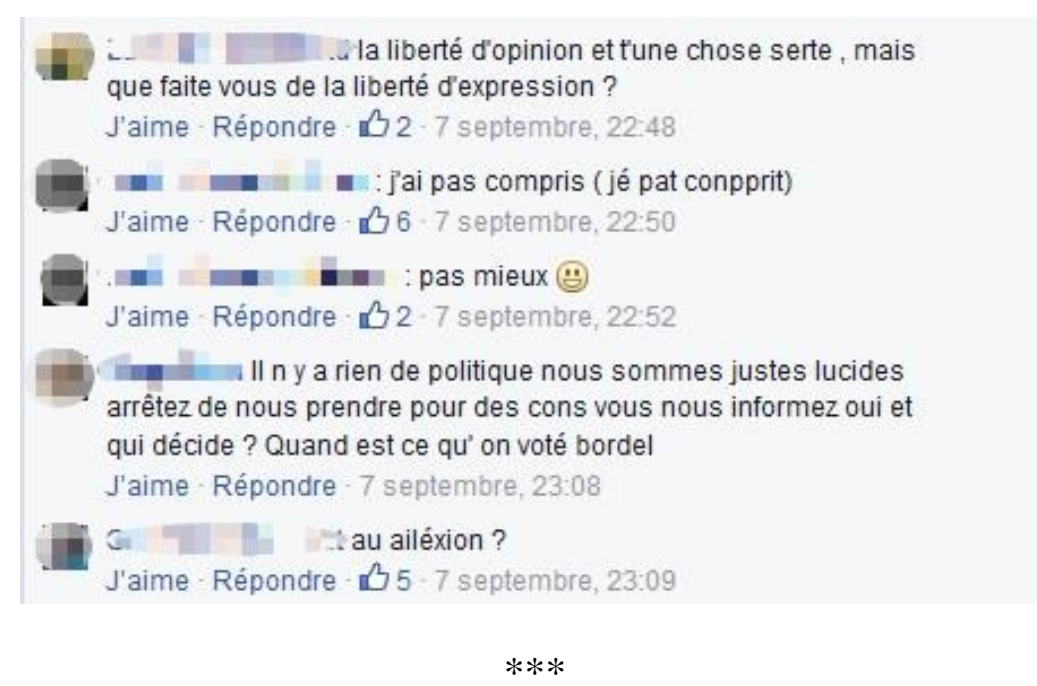

«La haine que les dominants portent aux dominés se manifeste ouvertement [...] dans les périodes de répression où la bourgeoisie se venge de sa peur » écrit Claude Grignon (1991, p. 8). On peut prolonger ses propos et affirmer que les dominants (ou les aspirants dominants) ne sont jamais aussi prompts à manifester leur mépris de classe que lorsque leur légitimité ne va plus de soi. Que l'on considère celui porté aux "cas soc'» ou aux «jeunesses économiques », il apparait en effet que la fragilisation dont fait l'objet la petite bourgeoisie culturelle lergnoise nourrit la domination qu'elle exerce (ou tente d'exercer) sur ses adversaires. Le mépris adressé aux membres de la Jeune Chambre Economique par les militants associatifs de la deuxième génération prend ainsi tout son sens à considérer la trajectoire déclinante des premiers et ascendante des seconds. Ces jeunes aux aspirations socio-résidentielles déçues, qui tentent de prendre la relève d'une petite bourgeoisie culturelle fragilisée, considèrent l'ascension de la Jeune Chambre Economique comme une usurpation scandaleuse d'une position qui aurait dû légitimement leur revenir. Le recours au registre du mépris de classe procède ainsi autant d'une tentative d'imposer aux membres de la petite bourgeoisie économique le sentiment de leur propre illégitimité que d'une volonté de marquer une ascension sociale à bien des égards fragilisée. De même, l'intensité du mépris voué aux classes populaires précarisées du centre-ville est à la mesure de l'ampleur de l'échec du travail de gentrification mené sur cet espace, qui concerne plus particulièrement les membres de la première génération qui y sont liés par la propriété. Ces voisins indésirables, incarnation du processus de déclin qui affecte les quartiers centraux, deviennent finalement le catalyseur de la frustration ressentie à vouer une partie de son existence et de ses ressources à un objectif qui s'impose au fil du temps comme irréalisable.

Ainsi, les fondements du mépris de classe ne sont pas forcément identiques selon les générations. Pour la première (née dans les années 1960 et 1970), il s'agit de défendre son inscription matérielle et symbolique dans l'espace, tandis que les membres de la seconde génération (nés dans les années 1980) cherchent davantage à marquer leur distance aux classes populaires, et par là même à leurs origines sociales. Dans les deux cas, le caractère inexorable que prend le processus de fragilisation qui s'exerce sur cette petite bourgeoisie culturelle fait que l'objectif est moins de disqualifier efficacement ses adversaires que de participer à la réassurance sociale du groupe. Cette forme particulière d'« autolégitimation » 
(Grignon et Passeron, 1989, p. 30) se présente alors comme un phénomène intrinsèquement collectif : elle est la conséquence des menaces qui pèsent sur la reproduction de la petite bourgeoisie culturelle lergnoise dans son ensemble, face auxquelles les manifestations du mépris de classe, qui se donnent toujours à voir au regard des autres membres du groupe, permettent de faire front.

AUST J and CRET B. 2012. L'État entre retrait et réinvestissement des territoires. Les Délégués régionaux à la recherche et à la technologie (DRRT). Revue française de sociologie, 53, 1, p. 3-33.

BEAUMONT A. 2017. Le pourboire et la classe. Genèses, 106, p. 94-114.

BIDOU-ZACHARIASEN C and POLTORAK J-F. 2008. Le «travail » de gentrification : les transformations sociologiques d'un quartier parisien populaire ». Espaces et sociétés, n² 132-133, 1, p. 107-124.

BILAND E. 2011. La fonction publique territoriale. Paris : France, La Découverte, 125 p.

BOURDIEU P. 1977. Sur le pouvoir symbolique. Annales, p. 405-411.

BOURDIEU P. 1979. La distinction : critique sociale du jugement. Paris : Éditions de Minuit, 670 p.

BOURDIEU P. 1993. La démission de l'État. In Bourdieu, P. La misère du monde. Paris : Seuil, p. 219-228.

BOURDIEU P. 1997. Méditations pascaliennes. Paris : Seuil, 316 p.

BOURDIEU P. 2016. Sociologie générale, vol. 2. Cours au Collège de France. Paris : Seuil, 1216 p.

BOZON M. 1982. La Mise en scène des différences. Ethnologie d'une petite ville de province. L'Homme, 22, 4, p. 63-76.

CAUCHI-DUVAL N, BEAL V and ROUSSEAU M. 2016. La décroissance urbaine en France : des villes sans politique. Espace populations sociétés, 2015/3-2016/1.

CAVENG R, DARBUS F, DENORD F, SERRE D and THINE S. 2018. Des morales de classe ?. Actes de la recherche en sciences sociales, 224, p. 76-101.

CHAMBOREDON J-C and LEMAIRE M. 1970. Proximité spatiale et distance sociale. Les grands ensembles et leur peuplement. Revue française de sociologie, 11, 1, p. 3-33.

CLERVAL A. 2008. Les anciennes cours réhabilitées des faubourgs : une forme de gentrification à Paris.

Espaces et sociétés, 132-133, p. 91-106.

COLLET A. 2015. Rester bourgeois : les quartiers populaires, nouveaux chantiers de la distinction. Paris : La Découverte, $282 \mathrm{p}$.

COULANGEON P. 2010. Sociologie des pratiques culturelles. Paris : La Découverte, 125 p.

CUSIN F. 2016. Y a-t-il un modèle de la ville française ?. Revue française de sociologie, Vol. 57, 1, p. 97-129.

DREYFUS F. 2011. La révision générale des politiques publiques, une conception néolibérale du rôle de l'Etat?. Revue française d'administration publique, 136, p. 857-864.

ELIAS N. 1985. Remarques sur le commérage. Actes de la recherche en sciences sociales, 60, 1, p. 23-29.

EPSTEIN R. 2011. Politiques de la ville: bilan et (absence de) perspectives ». Regards croisés sur l'économie, 1, p. 203-211.

EPSTEIN R. 2015. La gouvernance territoriale : une affaire d'État. La dimension verticale de la construction de l'action collective dans les territoires. L'Année sociologique, 65, 2, p. 457-482. 
FONDU Q and VERMERIE M. 2016. Les politiques culturelles : évolution et enjeux actuels. Informations sociales, 190, p. 57-63.

GOLLAC S and HUGREE C. 2015. Avoir trente ans dans le secteur public en 1982 et en 2002. Les transformations d'une filière de promotion sociale par le diplôme. Revue française d'administration publique, 153 , p. 23-43.

GRIGNON C. 1991. Racisme et racisme de classe. Critiques sociales, (2), p. 3-71.

GRIGNON C, COLLOVALD A, PUDAL B and SAWICKI F. 1991. Un savant et le populaire. Entretien avec Claude Grignon. Politix, 4, 13, p. 35-42.

GRIGNON C and PASSERON J-C. 1989. Le savant et le populaire : misérabilisme et populisme en sociologie et en littérature. Paris : Gallimard, Seuil, 260 p.

GUERAUT É. 2017. Quand les sociabilités numériques consolident les frontières sociales. Enquête sur le “milieu culturel” d'une ville moyenne. Sociologie, 8, 1-2017, p. 39-56.

GUERAUT É. 2018. Ascension et fragilisation d'une petite bourgeoisie culturelle. Une enquête ethnographique dans une ville moyenne en déclin. Thèse de doctorat, Paris, Paris Descartes, 519 p.

HELY M. 2009. Les métamorphoses du monde associatif. Paris : Presses universitaires de France, impr. 2009, xii+306 p.

LAFERTE G. 2015. Des études rurales à l'analyse des espaces sociaux localisés. Sociologie, 5, 4, p. 423-439.

LEGER J-F. 2011. L'évolution sociodémographique des villes moyennes de 1968 à 2006. Espace populations sociétés, 3, p. 557-576.

MATTINA C. 2004. Mutations des ressources clientélaires et construction des notabilités politiques à Marseille (1970-1990). Politix, nº 67, 3, p. 129-155.

MOULINIER P. 2016. Les politiques publiques de la culture en France, Paris : Presses universitaires de France (Que sais-je?).

NEGRIER E and TEILLET P. 2013. La réforme de l'État culturel local. Reconcentration instrumentale ou « contrôle orienté »?. Sciences de la société, 90, p. 76-91.

POUGET J. 2005. La Fonction publique : vers plus de diversité ?. France, portrait social, p. 143-162.

ROUBAN L. 2015. Les sommets de l'exécutif urbain : les maires des villes de plus de 30000 habitants entre 1983 et 2014. Revue française d'administration publique, 154, p. 377-390.

TISSOT S. 2011. De bons voisins : enquête dans un quartier de la bourgeoisie progressiste. Paris : Raisons d'agir, $313 \mathrm{p}$.

TISSOT S, GAUBERT C and LECHIEN M-H. 2005. Reconversions militantes. Limoges : Presses Universitaires de Limoges, 288 p.

URFALINO P. 2010. L'invention de la politique culturelle. Paris : Pluriel, 427 p.

VANDEBROECK D. 2015. Distinctions charnelles. Actes de la recherche en sciences sociales, 208, p. 14-39. 